\title{
Multi-scale analysis of neural activity in humans: implications for micro-scale electrocorticography
}

\author{
Spencer Kellis ${ }^{\mathrm{a}, 1}$, Larry Sorensen ${ }^{\mathrm{e}}$, Felix Darvas ${ }^{\mathrm{f}}$, Conor Sayres $^{\mathrm{f}}$, Kevin O’Neill III ${ }^{\mathrm{h}}$, Richard \\ Brown $^{\mathrm{a}, b, \mathrm{~d}}$, Paul House ${ }^{\mathrm{c}}$, Jeff Ojemann ${ }^{\mathrm{f}, \mathrm{g}}$, Bradley Greger ${ }^{\mathrm{h}}$
}

Departments of ${ }^{\mathrm{a}}$ Electrical and Computer Engineering, ${ }^{\mathrm{b}}$ Bioengineering, and ${ }^{\mathrm{c}}$ Neurosurgery, and the ${ }^{\mathrm{d}}$ School of Computing, University of Utah, Salt Lake City, UT, USA

Departments of ${ }^{\mathrm{e}} \mathrm{Physics}$, and ${ }^{\mathrm{f}}$ Neurological Surgery, ${ }^{\mathrm{g}}$ Center for Sensorimotor Neural Engineering University of Washington, Seattle, WA, USA

${ }^{\mathrm{h}}$ School of Biological \& Health Systems Engineering, Arizona State University, Tempe, AZ, USA

\section{"Corresponding author:}

E-mail: bradley.greger@asu.edu

${ }^{1}$ Present address: Division of Biology and Biological Engineering, California Institute of Technology, Pasadena, CA, USA 


\begin{abstract}
Objective: Electrocorticography grids have been used to study and diagnose neural pathophysiology for over 50 years, and recently have been used for various neural prosthetic applications. Here we provide evidence that micro-scale electrodes are better suited for studying cortical pathology and function, and for implementing neural prostheses.

Methods: This work compares dynamics in space, time, and frequency of cortical field potentials recorded by three types of electrodes: electrocorticographic (ECoG) electrodes, non-penetrating micro-ECoG $(\mu \mathrm{ECoG})$ electrodes that use microelectrodes and have tighter interelectrode spacing; and penetrating microelectrodes (MEA) that penetrate the cortex to record single- or multiunit activity (SUA or MUA) and local field potentials (LFP).

Results: While the finest spatial scales are found in LFPs recorded intracortically, we found that LFP recorded from $\mu \mathrm{ECoG}$ electrodes demonstrate scales of linear similarity (i.e., correlation, coherence, and phase) closer to the intracortical electrodes than the clinical ECoG electrodes.

Conclusions: We conclude that LFPs can be recorded intracortically and epicortically at finer scales than clinical ECoG electrodes are capable of capturing.

Significance: Recorded with appropriately scaled electrodes and grids, field potentials expose a more detailed representation of cortical network activity, enabling advanced analyses of cortical pathology and demanding applications such as brain-computer interfaces.
\end{abstract}

Keywords: Brain computer interface (BCI), human cerebral cortex, micro-electrocorticography grid, neural engineering, neural microtechnology. 


\section{Introduction}

The activity of the cerebral cortex may be recorded at a variety of scales and depths - from microelectrodes within the cortex to magnetic resonance imaging. Selecting an appropriate technology requires balancing a number of trade-offs including clinical responsibility to mitigate risk, signal integrity, cortical coverage, and specificity in time, space, or frequency. For example, electroencephalography may record brain potentials without any invasive procedure, but with less detail at a given cortical site than a penetrating microelectrode. Some applications, such as localization of epileptogenic zones, need coverage over large areas of cortex while others, like brain-computer interfaces, depend more on signal integrity and specificity. Understanding the properties of neural activity at various spatial and temporal scales and levels of invasiveness is important input for optimizing electrode array design for acquiring specific neural data.

\subsection{Signals and Dynamics}

Low-frequency (i.e., $<500 \mathrm{~Hz}$ ) intracortical, extracellular potentials are generated primarily by the sum of excitatory postsynaptic dendritic current sinks and sources near the recording electrode (Mitzdorf, 1985, 1987, Engel et al., 1990, Bullock, 1997, Buzsaki et al., 2012). Likewise, electrical potentials at the cortical surface consist principally of a summation of nearby synaptic dendritic activity (Freeman and Barrie, 2000), and are typically analyzed in the same or similar frequency range as LFPs (e.g., $<500 \mathrm{~Hz}$ ). Neuronal firing activity may bleed into the upper portion of the frequency range of either LFP or surface potentials, though likely with less power and specificity at the surface than within the cortex since the intracortical electrodes are closer to those small-amplitude sources. Because field potentials constitute a summation, synchronized oscillations will sum to larger contributions than asynchronous oscillations (Freeman and Barrie, 2000, Mehring et al., 2004, Ray et al., 2008). Also, since gray matter is a resistive medium within the frequency range of interest (Logothetis et al., 2007), there will be little or no capacitive filtering of these signals, and the frequency content of field potentials recorded intracortically should be similar to that recorded epicortically. Thus, for example, modulation of power in the gamma band (i.e., 30-80 Hz) coincident with motor activity is evident in both LFPs and surface potentials. However, the properties of electric fields would suggest that with increasing distance between the source and electrode, electric potentials will be smaller in magnitude and more diffuse in space.

In addition to passive propagation through the extracellular space, active processing by cortical circuits will play a role in defining spatiotemporal properties of field potentials. Synchronous oscillations are a fundamental property of the brain's processing mechanisms (Engel et al., 1990, Singer and Gray, 1995, von der Malsburg, 1995, Buzsaki and Draguhn, 2004, Fries, 2009). Temporally and spatially transient binding between and within populations of neurons will be reflected in dynamic and distributed correlations across cortex, if the signals are recorded with enough resolution in space and time. In some areas of the brain, such as in striate cortex, neurons with similar processing preferences have been shown to organize in cortical columns with diameters in the range of several hundred microns (Mountcastle, 1957, Singer and Gray, 1995). Interactions both within and between columns in visual cortex have demonstrated dynamic spatiotemporal patterns which are thought to represent the binding of features for cortical processing (Engel et al., 1990, Singer and Gray, 1995, von der Malsburg, 1995, Buzsaki and Draguhn, 2004, Fries, 2009, Ayzenshtat et al., 2010, Jia et al., 2011). Similar types of neuronal assemblies have been demonstrated in other areas of cortex (Georgopoulos et al., 1993, Amirikian and Georgopoulos, 2003), and subcentimeter organization has been observed in still other areas (Flinker et al., 2011). These assemblies comprise mesoscopic building blocks of cortical information processing and establish a lower limit on the actively-induced component of the field potentials' spatial extent on the order of a few hundred microns. It is of historical interest that the basic design of ECoG electrode grids instantiated in the 1950s has changed little since that time, while the evidence supporting mesoscopic information processing in the cerebral cortex has accumulated (Penfield and Jasper, 1954, Mountcastle, 1997).

The spatial extent of LFP has been characterized in a number of studies reporting a wide range of values, from a few hundred microns (Liu and Newsome, 2006, Katzner et al., 2009, Xing et al., 2009, Leski et al., 2013) to a millimeter or more (Engel et al., 1990, Kreiman et al., 2006, Berens et al., 2008, Jia et al., 2011, Leski et al., 2013, Seyedhosseini et al., 2014). These disparate results reflect the complexity inherent in continuous fields produced by the massive, intricate network of electrical sources and pathways in the cortex, and the complex way in which these elements interact to produce coherent outputs from myriad sensory and cognitive inputs (Linden et al., 2011). Indeed, the spatial properties of cortical field potentials have been shown to depend on frequency (Destexhe et al., 1999, Buzsaki and Draguhn, 2004, Liu and Newsome, 2006, Berens et al., 2008, Canolty and Knight, 2010, Leski et al., 2013), brain state (Destexhe et al., 1999), the nature of stimuli (Jia et al., 2011) and behavior (Fetz et al., 2000), across cortical layers (Xing et al., 2009, Linden et al., 2011, Leski et al., 2013), with anatomical location and between subjects (Aoki et al., 1999), and on neuron morphology, synapse distribution, and correlation in the synaptic activity (Linden et al., 2011, Leski et al., 2013).

The dynamic properties of recorded field potentials will also be influenced by the electrodes and electronics used to record them. When the voltages of two sensors are differentially amplified with respect to the same reference, active signals on the common reference could spuriously increase correlations between the amplified signals (Fein et al., 1988, Guevara et al., 2005). If two sensors record electric signals that originate from one or more common sources by volume conduction, those common signals will also increase the correlation between the two recorded signals. Finally, increased oscillatory synchronicity among neuronal populations recorded separately by the two sensors could also lead to higher correlations (Salinas and Sejnowski, 2001). Higher correlations are common to each of these scenarios; however, in the first case, the increase is an 
artefact of the recording setup, whereas in the latter two scenarios, the correlations reflect the underlying neural dynamics and the ability of the electrodes to capture those dynamics at a given spatiotemporal resolution. Recording artefact may be mitigated to a large extent by using a proper reference with high input-impedance amplifiers employing common-mode rejection as near the sensors as possible (Stacey et al., 2012, Stacey et al., 2013). Phase relationships between signals may be used to distinguish volume conduction (in which the phase is necessarily $0 \bmod \pi$ ) from other more physiologically-based correlations (Stam et al., 2007).

Although the true relationship between LFPs and ECoG may not be entirely understood, their similar underpinnings suggest that some information content may be shared and that some aspects of dynamic spatial and temporal scales may be similar. The principal advantage of intracortical LFP versus subdural ECoG has been better specificity of the intracortical field potentials; however, that comparison has typically been made between LFPs recorded on microelectrodes and ECoG recorded on relatively large disc electrodes. The discrepancy in electrode size is an important distinction, since broader synchronization would be required to effect change in the summed potential of larger neuronal populations integrated by the larger electrodes (Mehring et al., 2004, Ray et al., 2008, Slutzky et al., 2008, Slutzky et al., 2010).

There is strong evidence of fine spatial and temporal dynamics in cortical surface potentials (Bullock and McClune, 1989, Bullock et al., 1995b, 1995a, Menon et al., 1996, Towle et al., 1998, Shen et al., 1999, Wang et al., 2009, Gaona et al., 2011, Pei et al., 2011). Studies of electrocorticograms (ECoG) recorded by microelectrodes have demonstrated distinct spatial patterns within areas less than $1 \mathrm{~cm}^{2}$ (Wang et al., 2009, Kellis et al., 2010, Panagiotides et al., 2011). Coherence, a measure of correlation as a function of frequency, was found to decline with distance and frequency although abrupt changes were found even among nearest neighbors (within 5-10 mm) and over short timescales $(5-15 \mathrm{sec})$, and the distribution of coherence at any given separation distance could be large (Bullock et al., 1995a, Aoki et al., 1999, Shen et al., 1999). Coherence has been shown to differentiate function or state (Bullock et al., 1995a, Zaveri et al., 1999), pathological tissue (Towle et al., 1998), and anatomical location and cognitive tasks (Gaona et al., 2011). This growing body of work suggests that surface potentials may have better spatial and temporal resolution than has previously been available when recorded by grids of large electrodes. Recording at the appropriate spatial scale may therefore reveal fine-scale dynamics at the surface of the cortex.

\subsection{Electrodes}

Nonpenetrating microwire grids, also referred to as $\mu \mathrm{ECoG}$ grids, consisting of platinum microwires terminated to form a grid of contacts, have been used recently in a number of studies (Kellis et al., 2009, Leuthardt et al., 2009, Wang et al., 2009, Kellis et al., 2010, Mollazadeh et al., 2011, Panagiotides et al., 2011) (Fig. 1, left panel). The diameter of the microwire is typically less than $100 \mu \mathrm{m}$, and the grid pitch is typically less than 4 millimeters. $\mu$ ECoG grids are available from commercial manufacturers with FDA approvals for use in human subjects. Because the exposed area of a microwire is much smaller than that of a macro ECoG electrode, it will record from a smaller volume of cortex, and hence may be considered a more locally derived signal than standard ECoG.

The microelectrode array (MEA) (Nordhausen et al., 1994, Nordhausen et al., 1996, Maynard et al., 1997) (Fig. 1, middle panel) consists of a 10x10 grid of platinum-tipped silicon electrodes rising $1.5 \mathrm{~mm}$ out of a $4 \times 4 \mathrm{~mm}$ base ( $400 \mu \mathrm{m}$ pitch). Each electrode's tip is approximately $4 \mu \mathrm{m}$ in diameter. These arrays are inserted so that the electrode tips record the spiking activity of cortical neurons as well as continuous extracellular voltages within the cortex.

For this study, cortical field potentials were recorded from human subjects using clinical ECoG, $\mu$ ECoG electrodes, and the MEA. These data were explored to better understand the nature of the field potentials recorded at different scales and depths. Linear relationships in the data were estimated by averaging over substantial amounts of time in order to elicit persistent relationships; however, distributions contributing to these averages were also examined to illustrate the variability of the linear relationships. Signals tended to be more highly correlated the closer they were in space and time, and in the lower frequencies. Furthermore, surface potentials recorded at the microscale exhibited specificity near that of field potentials recorded intracortically. This work represents the first study of its kind to systematically compare the dynamics in space, time, and frequency of intracortical LFPs, subdural ECoG, and subdural $\mu \mathrm{ECoG}$ recorded in humans.

\section{Methods}

\subsection{Implants, Recordings, and Data selection}

All subjects in this study required surgical intervention to treat intractable epilepsy, and gave informed consent for their participation in an institutional review board-approved study. A 30-channel $\mu$ ECoG grid with 2-mm pitch was implanted in a male patient over the motor and somatosensory areas of cortex $(\mu \mathrm{ECoG} 1)$, and another grid of the same configuration was implanted in a third male patient over the motor cortex ( $\mu$ ECoG2). A 4x4 channel $\mu$ ECoG grid ( $\mu$ ECoG3) with 1-mm pitch was implanted in one male patient over the hand area of M1. In two other male patients, 96-channel MEAs were implanted in the right temporal lobe (MEA1) and right inferior frontal gyrus (MEA2). The MEA implant sites were regions of cortex targeted for subsequent resection. ECoG data were recorded from 64-channel, 8x8 clinical ECoG grids, with standard 2.3 mm exposed electrode area and 1-cm spacing (ECoG1, ECoG2). 
Data from the $\mu \mathrm{ECoG}$ grids and MEAs were recorded at 10,000 or 30,000 samples per second (Cerebus System, Blackrock Microsystems, SLC, UT). The noise floor of the Cerebus recording system is $\sim 2 \mu \mathrm{V}$. The $\mu \mathrm{ECoG}$ data were recorded against the clinical reference, located several centimeters away in the epidural space. The MEA data were referenced to $2 \mathrm{~cm}$ exposed length of low-impedance wire which was either in the subdural or epidural space. The $\mu \mathrm{ECoG}$ and MEA recordings were amplified at the head stage with common-mode rejection (CMR) to minimize contamination from the reference in the recorded neural data. Data from the clinical ECoG grid were recorded at 1,000 or 10,000 samples per second with a reference located in the epidural space.

The impedance of the MEA electrodes was measured in vitro prior to implantation (MEA1 $=311 \pm 154 \mathrm{k} \Omega$; MEA2 $=$ $398 \pm 163 \mathrm{k} \Omega$ ) and in vivo (MEA1 $=869 \pm 342 \mathrm{k} \Omega$; MEA2 $=2254 \pm 781 \mathrm{k} \Omega$ ). It was not possible to measure impedance for the $\mu \mathrm{ECoG}$ or ECoG electrodes prior to implantation because of sterilization constraints, or in vivo due to limitations of the hardware. However, impedance characteristics of $\mu \mathrm{ECoG}$ grids with the same materials and from the same manufacturers have been examined previously (Kellis et al., 2011b). Representative impedance data from both $\mu \mathrm{ECoG}$ and ECoG electrodes were collected in vitro $(\mu \mathrm{ECoG}=228 \pm 139 \mathrm{M} \Omega ; \mathrm{ECoG}=600 \pm 134 \Omega)$. The input impedance of the recording amplifiers used for $\mu \mathrm{ECoG}$ and MEA recordings $\left(\sim 10^{13} \mathrm{Ohms}\right)$ was significantly higher than both the recording electrode impedance $\left(\sim 10^{2}-10^{5}\right.$ Ohms) and the reference electrodes $\left(\sim 10^{2} \mathrm{Ohms}\right)$.

Continuous segments of $1000 \mathrm{sec}$ were selected from recordings made with each electrode grid or array (648 sec for ECoG2). Where available, the clinical video feed was reviewed to ensure that the patient was awake and behaving naturally (although all patients were confined to the hospital beds while in the Neurocritical Care Unit); otherwise, the data were selected from recordings between the hours of $10 \mathrm{am}$ and $6 \mathrm{pm}$, but not during any experimental sessions, to maximize the likelihood of awake natural behavior. The data were reviewed to ensure no seizures or other significant problems were present.

Prior to analysis, the data with sampling rates above 1,000 samples per second were low pass filtered (Butterworth filter, order 14, passband $400 \mathrm{~Hz}$, stopband $500 \mathrm{~Hz}$ ) then downsampled to 1,000 samples per second. All filtering was performed both in the forward and reverse directions to avoid phase distortion. Due to contamination from line noise, the data were further processed to attenuate noise (Chronux package; fitting sine waves to data using overlapping windows) (http://chronux.org) (Mitra and Bokil, 2008).

\subsection{Correlation Functions}

Correlation functions were estimated for all possible two-channel permutations (including autocorrelation). For two time series $x$ and $y$ with means $\mu$ and standard deviations $\sigma$, the normalized correlation coefficient $\rho_{x y}$ between them was calculated over a range of lags $m$ as

$$
\rho_{x y}(m)=\left\{\begin{array}{rr}
\frac{\sum_{n=0}^{N-|m|-1}\left(x_{n+m}-\mu_{x}\right)\left(y_{n}-\mu_{y}\right)}{\sigma_{x} \sigma_{y}}, & m \geq 0 \\
\rho_{x y}^{*}(-m), & m<0
\end{array}\right.
$$

These calculations were performed for nonoverlapping 2-second data segments, for $-2 \mathrm{sec}<\mathrm{m}<2 \mathrm{sec}$. Then, the crosscorrelation function (CCF) between each pair of channels was estimated by averaging results across all segments.

Zero-lag correlation coefficients, i.e., $\rho_{\mathrm{xy}}(0)$, were used to form estimates of correlation versus distance. These values were calculated for each 2-sec segment and for each pair of channels, then averaged for each unique separation distance. Separately, zero-lag correlation between each pair of channels was also calculated using all $1000 \mathrm{sec}$ of data simultaneously.

\subsection{Coherence Analysis}

Coherence is a normalized measure of the linear relationships between two time series as a function of frequency. For two time series $x_{1}(t)$ and $x_{2}(t)$, given estimates of their power spectra $S_{1}(f)$ and $S_{2}(f)$, and where * denotes complex conjugation, the coherence between them is given by:

$$
C_{1,2}(f)=\frac{S_{1}^{*}(f) S_{2}(f)}{\sqrt{S_{1}^{*}(f) S_{1}(f)} \sqrt{S_{2}^{*}(f) S_{2}(f)}}
$$

This relationship normalizes the cross power spectral density (PSD) by the individual PSDs, and provides a measure of the correlative strength between the two signals in each frequency band. Only the magnitude of the coherence, $\left|\mathrm{C}_{1,2}(\mathrm{f})\right|$, is reported in this study.

The Chronux package was used to calculate multitaper estimates of the power spectra in each 2-sec segment, with a time-bandwidth parameter of 2, 3 tapers, 2048-point FFTs, and average the results over all segments. These results were further averaged over all channel pairs at each unique separation distance, to form an estimate of coherence as a function of both frequency and separation. For each frequency, the half-height of the coherence-versus-distance function, with units of distance, 
was calculated as the separation at which the coherence had declined midway between the maximum and minimum values. Because the separation distances were sparsely sampled, a cube smoothing spline was applied to the coherence versus distance points and the half-height values were estimated from the smoothed data. The smoothing was applied using the MATLAB "fit" function (The MathWorks, Inc., Natick, MA, USA).

\subsection{Phase Analysis}

Given a signal $x(t)$ and its Hilbert transform $\tilde{x}(t)$, the instantaneous phase $\phi(t)$ of the signal may be calculated as the phase angle of the analytic signal $z(t)=x(t)+i \tilde{x}(t)$ :

$$
\phi(t)=\arctan \left(\frac{\tilde{x}(t)}{x(t)}\right)
$$

Two signals are phase-locked if their instantaneous phase difference $\Delta \phi(t)=\phi_{1}(t)-\phi_{2}(t)$ is concentrated around a specific value $\phi_{\text {lock }}$. The extent to which two signals are coupled can be evaluated by calculating the magnitude of the average projection of their instantaneous phase differences on the unit circle (Stam et al., 2007):

$$
R=\left\langle e^{i \Delta \phi}\right\rangle=\left|\frac{1}{N} \sum_{k=1}^{N} e^{i \Delta \phi\left(t_{k}\right)}\right|
$$

If $\Delta \phi\left(t_{k}\right)=\phi_{\text {lock }} \forall k$, the signals are perfectly phase locked and $R=1$; if the phase differences are uniformly randomly distributed, $R=0$. However, $R>0$ cannot distinguish between phase locking due to volume conduction, where $\phi_{\text {lock }}=0 \bmod \pi$, and phase locking due to other phenomenon.

Phase Lag Index (PLI) is a measure of the asymmetry in the distribution of instantaneous phase difference between two signals (Stam et al. 2007). Such asymmetry requires that one signal consistently lags in time behind the other and excludes the possibility of volume conduction or a signal on the reference electrode. PLI may be calculated as:

$$
P L I=\mid\langle\operatorname{sign}(\Delta \phi(t)\rangle|
$$

The phase lag index takes values in $[0,1]$. When $P L I=1$, the signals are perfectly phase locked at $\phi_{\text {lock }}=\langle\Delta \phi(t)\rangle$ from $0 \bmod \pi$. When $P L I=0$, the signals are non-coupled, or coupled with a phase difference centered around $0 \bmod \pi$.

Together, $R$ and $P L I$ can be used to characterize the nature of the phase relationship between two signals. Although these quantities are used in some cases to evaluate functional connectivity, for this work their purpose is to determine whether the recorded neural data are caused by volume conduction (or, equivalently in terms of phase, contamination from a signal on the reference electrode), or whether the measured correlations are a consequence of spatiotemporal dynamics recorded in neural tissue. Under this constraint, it is sufficient to show either low $P L I$ and low $R$ (i.e. random distribution of phase differences) or high $P L I$ and high $R$ (i.e. concentration of phase differences around a value other than 0 -mod- $\pi$ ).

Both $R$ and $P L I$ were calculated for the broadband neural data in each of the two-second long segments. To identify whether these results were significantly larger than zero, both $R$ and $P L I$ were also calculated for phase-randomized surrogate data: for each segment, 100 surrogates were created by applying a random circular shift (uniformly selected in the range $0.5-1.0$ sec) to one of the channels. For each channel pair, the Kruskal-Wallis nonparametric one-way ANOVA with the Tukey-Kramer post-hoc test was used to ascertain whether the mean of the real values (calculated for each two-second segment) was significantly different from mean of the phase-randomized surrogate values (100 values calculated for each two-second segment) at the 95\% confidence level. To characterize estimates of the grand mean of $R$ and $P L I$ for each grid, data were combined across segments and channel pairs (all real values in one set, and all surrogate values in a second set), and a bias corrected percentile bootstrap with 1000 samples was used to calculate the confidence interval at the $95 \%$ confidence level. To characterize estimates of the mean $R$ and $P L I$ at each separation distance, all electrode pairs with equal separation distance were combined, and a bootstrap with 1000 samples was used to calculate confidence intervals at the $95 \%$ confidence level.

\section{Results}

\subsection{Correlations}

Correlations estimated from data recorded with each grid revealed patterns in time and space in the neural signals (Fig. 2, Suppl. Fig. S1). To elicit persistent relationships in the neural signals, correlations were averaged over time (500 2-sec segments), and at each unique separation distance. Correlations were strongest for signals close in both space and time, and departure in either dimension resulted in decreasing correlation. In addition to these general trends, dynamic activity was also observed in both time and space. 
CCFs exhibited reduced magnitude with increasing separation until reaching a baseline, after which they did not change substantially with further increases in separation distance. For example, after a few incremental separations (about $30 \mathrm{~mm}$ ), cross-correlations in the ECoG grid were negligibly small at all lags. CCFs from the $\mu \mathrm{ECoG}$ grids reached their baseline correlational value within approximately 4-6 mm, whereas CCFs from the MEAs exhibited an inflection point and subsequent baseline by approximately $2 \mathrm{~mm}$ separation.

The relationship between correlation and distance is explored in more detail in Fig. 3. In the top panel, the mean value of the correlation at each unique separation distance is shown for all data sets. Correlations fall more quickly to their baseline values for $\mu \mathrm{ECoG}$ than for ECoG, but not as fast as the MEA. Only the ECoG grid fell to a negligibly small correlation, about 0.1 , and remained there after the inflection point around $30 \mathrm{~mm}$. The MEAs and $\mu E C o G$ grids, whose maximum separation distance is much smaller than that of the ECoG grids, demonstrated higher correlations at their largest separations. The lower three panels of Fig. 3 shows the distribution of correlations at each separation distance for each data set, illustrating that the correlation at a given distance can be variable among the different electrode pairs.

To investigate the nature of correlations between individual pairs of channel in more detail, the correlation of each channel with all other channels in the same grid are shown in Suppl. Fig. S2. These correlations were calculated using the entire time series simultaneously $(1000 \mathrm{sec})$. Here again, the general trend shows decreasing correlation with increasing distance. However, there were some widely separated channel pairs with high correlations. Correlations could fluctuate rapidly as the distant electrode was moved a few millimeters, or as time progressed a few seconds. These observations illustrate the dynamic nature of correlation at separated cortical sites as well as dynamic nature of correlations over time.

\subsection{Coherence: The Role of Frequency}

Coherence, a measure of linear similarity as a function of frequency, demonstrated patterns in frequency and space (Fig. 4, Suppl. Fig. S3). Coherence functions were estimated by averaging the results of consecutive 2-second segments (500 2-sec segments). Coherence was generally strongest for signals close in space and in lower frequencies, and generally weakest in the high frequencies. This observation is illustrated in Suppl. Fig. S4, which visualizes coherence across each grid in a low frequency band, $8-12 \mathrm{~Hz}$, and a high frequency band, 30-80 Hz, side-by-side. The ECoG grid had the smallest maximum coherence (discounting auto-coherence and peaks due to line noise contamination) of any of the grids.

For the MEAs and $\mu \mathrm{ECoG}$ grids, coherence declined more rapidly with distance in higher frequencies than it did in lower frequencies. To characterize this behavior, the distance required for the coherence to decay to half its maximum range was calculated for each frequency (Fig. 5). This quantity, called half-height decay (HHD), is a measure of the spatial extent of the signals. Large HHD suggests that the signal remains highly correlated between widely separated electrodes; small HHD indicates that correlation declines quickly with separation. Coherence in the beta band, which typically shows high correlations over large spatial area, was highest in all grids. The ECoG grid had the largest HHD across all frequencies, with a peak of $8.45 \mathrm{~mm}$ at 6.84 $\mathrm{Hz}$ and a baseline value of $5.0 \mathrm{~mm}$ average between $300-400 \mathrm{~Hz}$. The $\mu \mathrm{ECoG}$ grids exhibited variable peak HHD with values ranging from $2.13 \mathrm{~mm}$ to $3.45 \mathrm{~mm}$; however, HHD was tightly grouped in the high frequencies across the $\mu \mathrm{ECoG}$ grids, with average values in the range $0.69-0.87 \mathrm{~mm}$ between $300-400 \mathrm{~Hz}$. Baseline HHD was smallest for the MEAs, at $0.16 \mathrm{~mm}$ for both MEA1 and MEA2. These results demonstrate that cortical surface potentials contain millimeter and submillimeter scale dynamics.

\subsection{Phase Relationships}

Phase analysis indicated the data were free of significant volume conduction or active reference contamination. Instantaneous phase differences were either significantly concentrated (high $R$ ) away from 0-mod- $\pi$ (high $P L I$ ), or not significantly concentrated around any value (low $R$ and low $P L I$ ) for nearly all electrode pairs examined in this study (Fig. 6). The microelectrode data (both MEA and $\mathrm{uECoG}$ ) exhibited the highest percentages of concentrated, non 0-mod- $\pi$ phase differences (MEA1: 95\%, 4346/4560; MEA2: 97\%, 4419/4560; $\mu$ ECoG1: 88\%, 384/435; $\mu$ ECoG2: 94\%, 410/435; $\mu$ ECoG3: 100\%, $120 / 120$, of channels had both $R$ and $P L I$ significantly above the surrogate level with $\mathrm{p}<0.05$ ), whereas just $23 \%, 454 / 2016$ of ECoG electrode pairs demonstrated the same result. However, an additional 15 electrode pairs for $\mu \mathrm{ECoG} 2$ and 879 electrode pairs for ECoG had neither $R$ nor $P L I$ significantly above the surrogate level, indicating that the instantaneous phase differences were not concentrated around any value including $0 \bmod \pi$.

In addition to exhibiting either random phase differences or phase differences concentrated away from 0 mod $\pi$, both $R$ and PLI showed dependence on separation distance between electrode pairs for all grids (Fig. 7). In each grid, these two quantities decreased toward the phase-randomized surrogate level as the distance separating electrode pairs increased. In the ECoG dataset, the average $P L I$ and $R$ fell to the surrogate level by approximately $20 \mathrm{~mm}$, suggesting a lack of any consistent phase relationship between electrode pairs at these large separation distances. Both $P L I$ and $R$ were flat between 3 and $4 \mathrm{~mm}$ separation for both UEA1 and UEA2, although with larger confidence intervals due to fewer channel pairs contributing to the average. The $\mu \mathrm{ECoG}$ data also exhibited decreasing $P L I$ and $R$ with increasing distance, but did so more gradually than UEA1 or UEA2. 
In two datasets (UEA2 and uECoG2), the $95 \%$ confidence intervals between the phase-randomized surrogate and real $P L I$ calculations overlap at the largest separations (UEA2: $>4 \mathrm{~mm} ; \mu \mathrm{ECoG} 2:>12 \mathrm{~mm}$ ), while $R$ remains significantly above the surrogate level at the same distances. (In the ECoG dataset, both $R$ and PLI fall to the surrogate level by the same separation.) The lack of statistical support for non 0 mod $\pi$ phase lags means that the source of correlations at these large separation distances is unclear for these two datasets. It is important to note that only a small percentage of channel pairs contribute to the estimates at these large separations. However, correlations at these separations could represent a baseline value caused by volume conduction or other contamination, from which deviations might represent more active synchronization among nearer neural populations. If this is true, the minimum value would represent a constant shift across separation distances, but should not change the spatial dependencies in either correlation or coherence.

\section{Discussion}

Linear measures, i.e., correlation and coherence, have been used to evaluate temporal, spatial, and frequency-domain dynamics in neural signals recorded intracortically by microelectrodes and epicortically by both micro- and macroelectrodes. Correlation and coherence were strongest across all modalities for signals closest in space and time, and in the lower frequencies.

Furthermore, these linear relationships, on average, tended to decay gradually over space, time, and frequency, but significant dynamics remained even at large separations. The baseline half-height decay of the signals, i.e., the average HHD between 300$400 \mathrm{~Hz}$, was $160 \mu \mathrm{m}$ for the MEAs, $690-870 \mu \mathrm{m}$ for the $\mu \mathrm{ECoG}$ grids, and $5.0 \mathrm{~mm}$ for the ECoG grids. These data suggest that intracortical LFP is the most specific method, in both space and time, of recording cortical field potentials. Furthermore, surface potentials recorded at the appropriate scale, i.e. by microelectrodes, contain fine dynamics in both space and time which are comparable in extent to the intracortical LFP and with greater spatiotemporal resolution than ECoG electrode grids.

\subsection{Factors Influencing Spatiotemporal Dynamics}

The resolution with which neural activity may be recorded at any depth is practically limited by a number of factors. The distance between the electrode and the neural source(s) and the impedance characteristics of the materials and structure in the intervening space determine the severity of the attenuation and filtering of the neural activity before it can be recorded. The size of the electrode and its impedance properties relative to both the surrounding tissue and the amplifier input influence how much of the surrounding tissue will contribute to the electric potential (Slutzky et al., 2010, Wodlinger et al., 2011, Stacey et al., 2012). Small, transient dynamics, which would be masked in the integration of larger volumes of cortex, might be observable when recorded by tightly spaced microelectrodes. Intuitively, it is reasonable to expect that field potentials recorded by microelectrodes within the cortex should afford the greatest resolution since they are nearest the signal generators. These results support such a possibility, in that the MEAs demonstrated, on average, the smallest half-height decays in both time and space. Such concepts also suggest that $\mu \mathrm{ECoG}$ may consist of a fundamentally different signal than traditional ECoG, an idea further supported by the different spatiotemporal properties found for the $\mu \mathrm{ECoG}$ vs. the ECoG grids.

Processing mechanisms of the cortex, including differences across cortical layers, have been shown to influence the spatial extent of LFP. For example, it has been observed in cat suprasylvian cortex that coherence falls off more rapidly with distance during wake or REM sleep states than during slow wave sleep (Destexhe et al., 1999). Sleep or drowsiness most typically lowered coherence across frequencies in human cortex (Bullock et al., 1995a), and alpha rhythms demonstrated significantly different coherence when resting with eyes open vs. squeezing a sponge with eyes closed (Zaveri et al., 1999). Spatially extensive coherence has been observed in macaque V1 with large-grating visual stimuli whereas smaller stimuli were found to induce more local activity (Jia et al., 2011), and long-distance correlations (e.g., between pre- and post-central LFPs) have been shown to be more likely during exploratory tasks than during trained motor movements (Fetz et al., 2000). The cortical spread of LFP was found to vary significantly across cortical layers in macaque V1, from $150 \mu \mathrm{m}$ in layer 4B to $280 \mu \mathrm{m}$ in layer 2/3 (Xing et al., 2009). For comparison, correlations in spontaneous neuronal spiking activity in cat visual cortex have been shown to fall to $50 \%$ within $240 \mu \mathrm{m}$ (Ch'ng and Reid, 2010).

Because results were calculated by averaging over large amounts of neural data recorded during natural behavior and were free of seizure activity, it is unlikely that observed correlations could be the result of behavior- or task-related neural processing, which would have had to persist over 1,000 seconds. Thus, we believe that the dynamics described in this study illustrate practical recording characteristics of MEA, $\mu \mathrm{ECoG}$, and ECoG electrodes. However, it is likely that brain state, behavior, or pathology (e.g. seizure activity) could significantly impact the correlation, coherence, and phase relationship metrics observed here; and could serve as markers for different conditions of the underlying neural networks.

The existence of cortical columns has been supported by many studies using a variety of techniques, however, cortical columns have complex and differing forms that vary with the particular cortical areas (Rakic, 1988, 2008). The cortical areas form into complex hierarchies with other cortical areas and sub-cortical structures (Alexander et al., 1986, Felleman and Van Essen, 1991, Kelly and Strick, 2003). Because clinical need mandated the anatomical location of electrodes for all data used in this study, it was not possible to control the placement of electrodes at different cortical areas, e.g. primary sensory-motor cortex of the parietal and frontal lobes versus tertiary cortex of the frontal and temporal lobes. Due to differing cytoarchitecture, cortical and sub-cortical connectivity, and cortical columnar structure of these cortical areas, the placement of electrode grids and arrays at different cortical areas may have affected the observed spatiotemporal dynamics of the field potentials. 


\subsection{Characteristics of Spatiotemporal Dynamics}

Figures demonstrating correlation versus distance were characterized by an inflection point where the curve began to form a horizontal asymptote (except in the case of $\mu \mathrm{ECoG} 2$, which was limited by the number of channels and the largest available channel spacing). The presence of a horizontal asymptote suggests that there is a baseline correlation in the field potentials. In the case of the ECoG grid, the formation of a horizontal asymptote began between 20 and $30 \mathrm{~mm}$, and the baseline correlation was close to 0 . For the MEAs and $\mu \mathrm{ECoG}$ grids, the correlation began to asymptote at higher levels, with the MEA baseline lower than the $\mu \mathrm{ECoG}$ baseline. It is possible that the high baseline correlation in the small grids and the lack of an obvious horizontal asymptote in $\mu \mathrm{ECoG} 2$ is due to the small size of the grids, or particularly in the case of $\mu \mathrm{ECoG} 2$, it could also be due to the few unique separations between channels. However, it could also be the case that intracortical LFP is more dynamic on finer scales than surface potentials regardless of the size of the recording electrode.

In some cases, high correlations were present between electrodes which were separated by large distances, even when both $P L I$ and $R$ were both significantly high. Spatially distinct correlations could arise when one region of cortex is functionally bound to a discretely separate region of cortex during task or stimulus (Aoki et al., 1999, Shen et al., 1999). Such synchronous oscillations in the field potentials of the two regions would be registered as an increase in the level of correlation between them. In further evidence that the underlying brain activity was spatially variable, the distributions of correlation versus distance over space and time (Fig. 3) was dynamic over time, but changes in spatial correlation patterns demonstrated even larger distributions (Shen et al., 1999).

Clear changes were evident in the strength of linear relationships between channels across frequency. Previous work has found similar dependence on frequency: tuning for speed and direction in extrastriate cortex (MT) was found to exhibit superior specificity for high frequencies, i.e. gamma and above (Liu and Newsome, 2006). In general, high frequencies have been shown to exhibit more local extent whereas low frequencies are more spread out (Destexhe et al., 1999, Buzsaki and Draguhn, 2004, Liu and Newsome, 2006, Berens et al., 2008, Canolty and Knight, 2010). It has been suggested that this dependence on frequency could be an artifact of low pass filtering by capacitive properties of cortical tissue (Bedard et al., 2004); however, with direct experimental evidence for isotropically flat frequency response in gray matter, the impedance of cortical tissue is unlikely to be a significant factor in any frequency-dependent trends in the decay of neural activity (Logothetis et al., 2007). A different plausible explanation for the dependence of correlation on frequency is that small, local populations with fast transient activities generate high frequency oscillations whereas low frequency content represents spatially broad modulation of local population activity as these distributed processing elements are coordinated to produce coherent, cognitive outputs (Buzsaki and Draguhn, 2004, Fries, 2009, Canolty and Knight, 2010).

The ECoG grid had the smallest maximum coherence, discounting auto-coherence or noisy peaks, of any of the grids. It is possible that the dynamics seen in the other grids were largely missed in the $10 \mathrm{~mm}$ separating the closest ECoG electrodes; the smaller grids had much larger coherence at the closest spacing although the high frequencies exhibited small coherence across all separations.

\subsection{Active References and Common Sources}

That the signals recorded on microelectrodes originate from local, discrete sources was supported by the inverse relationship between separation distance and measures of correlation and coherence, which otherwise would have been much flatter with large absolute values (Fig. 2 and 4; Suppl. Fig. S1 and S3). In particular, coherence in moderate and high frequencies was quite low. Additionally, the analysis of the instantaneous phase differences between all electrode pairs indicated that the signals recorded by microelectrodes were largely driven by local, discrete sources, e.g. cortical columns, and not by volume conduction or a signal on the reference electrode (Fig. 6 and 7). The inverse spatial dependence for both the phase lag index PLI and the phase lag concentration quantity R suggests that the most consistent phase differences exist between nearby electrodes. In the context of this work, such a finding is useful because it could indicate opportunities to adapt electrode size and/or spacing to the resolution of the underlying neural source dynamics. All of the results in this work are based on the data recorded through high input-impedance recording amplifiers with common-mode rejection that mitigates the potential effects of a signal on the low input-impedance reference electrode. Together, these observations suggest that the dynamics in space, time, and frequency described in this work are representative of the capabilities of each electrode type to capture the dynamics of the neural activity.

\subsection{Limitations}

The data collected for this study were acquired in patients undergoing surgical intervention for medically refractory epilepsy. At least one study has illustrated that pathology in cortical tissue affects the coherence (Towle et al., 1998); thus, the results presented here must be taken in that context. In particular, the MEAs were implanted in portions of the temporal lobe likely to be resected and may have been impacted by pathological cortical network interactions. Portions of the ECoG are likely to be over epileptogenic zone, while the $\mu \mathrm{ECoG}$ grids were generally placed away from epileptogenic zones and over functional areas 
which could be exercised with motor and speech tasks. Although the possibility of contamination of the data by epileptiform discharges is real, care was taken to select data which was not recorded during seizure and which was free of interictal spiking.

There are many improvements remaining to be made in the design and manufacture of micro-ECoG electrodes.

Previous work has suggested that current-generation $\mu \mathrm{ECoG}$ grids, which are often stiff and do not conform well to the cortical surface, may allow cerebrospinal fluid (CSF) to rest between the grid and cortical surface (Kellis et al., 2011a). Such a layer would allow conductive paths between electrodes which would degrade the spatiotemporal detail in signals recorded by the electrodes. Analytical models have also suggested that current-generation $\mu \mathrm{ECoG}$ electrodes are so small that their sensitivity to important depths in cortex is substantially degraded (Wodlinger et al., 2011). These elements illustrate the significant challenges in designing an appropriate electrodes for recording cortical surface potentials: while spatial design variables such as electrode size and spacing are important, other design aspects such as conformity and thickness also play a crucial role (Kim et al., 2010). Spatiotemporal characteristics of recorded surface potentials may improve substantially with improvements in the design and manufacture of the electrodes and proper integration into data acquisition systems (Stacey et al., 2012, Stacey et al., 2013).

\section{Conclusion}

The brain is comprised of many billions of distinct sources, which operate at the scale of microns and milliseconds, and are organized into a complex hierarchy of networks. Selecting an electrode to record activity generated by this complex network requires balancing a number of factors including patient risk, cortical coverage, and spatiotemporal specificity required by the application. This work has shown that surface potentials recorded by microelectrodes demonstrate fine spatiotemporal resolution - similar to that recorded intracortically and much finer than when the same potentials are recorded by macro ECoG electrodes. Thus, micro-electrocorticography both at the cortical surface and intra-cortically presents an attractive option for applications like brain-computer interfaces and the study and diagnosis of neural pathology (Schevon et al., 2008, Brinkmann et al., 2009, Hwang and Andersen, 2009, Kellis et al., 2009, Leuthardt et al., 2009, Schevon et al., 2009, Wang et al., 2009, Waziri et al., 2009, Kellis et al., 2010, Schevon et al., 2010, Stead et al., 2010, Bansal et al., 2011, Kellis et al., 2011a, Bansal et al., 2012, Hwang and Andersen, 2012, Chestek et al., 2013), likely due to the matching of the scale of the sensors to the scale of cortical columns that act as fundamental computation units of information processing in the cerebral cortex. 


\section{Acknowledgements}

This work was supported by a Utah Research Foundation grant and DARPA N66001-06-C-8005 funding (BG); and the Engineering Research Centers Program of the National Science Foundation under award number EEC-9986866 (RB). The authors thank Kristin Kraus for her editorial assistance, the Univ. of Utah Health Sciences EEG staff for their assistance in conducting the study, and most importantly the patients for participating in the study. 


\section{Figure Legends}

\section{Figure 1.}

Images of the types of devices used to record neural activity for this study. (A) A penetrating microelectrode array (gold wires leading to black square) and surface $\mu \mathrm{ECoG}$ grid (green wires leading to translucent circle) shown implanted alongside standard electrocorticographic (ECoG) electrodes (silver disks with numbers overlaid). (B) Electrode locations co-registered between MRI and CT scan of (A): the red dots indicate ECoG electrodes, the yellow square indicates position of MEA1, and green circle indicates location of a $\mu \mathrm{ECoG}$ grid.

\section{Figure 2.}

Correlation functions estimated for each grid type. The left panel in each row shows correlations plotted against lag, with color representing separation distance and the inset depicting the data from 0.0 to $1.0 \mathrm{sec}$ logarithmically. The middle panel shows the correlations plotted against separation distance, with color representing lag. The right panel is a mesh showing the correlations plotted against both separation distance and lag. The mesh is log-spaced in the case of lag and linearly spaced in the case of separation distance. The top row shows correlations from the ECoG1 grid; the middle row shows correlations from $\mu \mathrm{ECoG1}$; and the bottom row shows correlations from MEA1. See Suppl. Fig. S1 for the other grids.

\section{Figure 3.}

Correlation vs. distance for each grid type. For each of the grids, correlations were measured between all pairs of channels for consecutive non-overlapping two second segments of data. Colors are consistent through the figure, with black and grey indicating the ECoG grids; red and orange hues indicating the $\mu \mathrm{ECoG}$ grids; and blues indicating the MEAs. The top panel shows the average correlation at each unique separation distance separating pairs of channels for all three grid types simultaneously. These values include averaging over consecutive segments of data (i.e., averaging over time), as well as averaging over the channel pairs of a given separation distance (i.e., averaging over space). Because of the different physical scales of the three grids, the lower three panels show the distribution of values (box $=25^{\text {th }}$ to $75^{\text {th }}$ percentiles, whiskers $=$ extrema, points $=$ outliers) at each unique separation distance for the three grid types, with the average for each of the electrode grids drawn as a thick line.

\section{Figure 4.}

Coherence functions estimated for each grid type. The left panel in each row shows coherence plotted against frequency, with color representing separation distance. Coherence values for frequencies contaminated by line noise, e.g., $60 \mathrm{~Hz}$ and harmonics, are left blank. The middle panel shows the coherence plotted against separation distance, with color representing frequency. In these panels, coherence was averaged over frequencies to produce estimates of the coherence within the standard electroencephalographic frequency bands: delta $(0-4 \mathrm{~Hz})$, theta $(4-8 \mathrm{~Hz})$, alpha $(8-12 \mathrm{~Hz})$, beta $(12-30 \mathrm{~Hz})$, gamma $(30-80 \mathrm{~Hz})$, chi $(80-200 \mathrm{~Hz})$, and a high band $(200-400 \mathrm{~Hz})$. The right panel is a mesh showing the coherence plotted against both separation distance and frequency. The mesh is log-spaced in the case of frequency and linearly spaced in the case of separation distance. The top row shows coherence from the ECoG1 grid; the middle row shows coherence from $\mu E C o G 1$; and the bottom row shows coherence from MEA1. See Suppl. Fig. S3 for the other grids.

\section{Figure 5.}

Half-height decay (HHD) of the coherence (in millimeters). The half-height decay quantifies how far apart two electrodes must be to reduce their coherence by half. These results are shown as a function of frequency, for each grid in the study. For each frequency, the coherence from all channel pairs at a given separation distance were averaged to find coherence as a function of distance. A cubic smoothing spline was fit to this curve, and the distance in millimeters found at which the coherence had dropped to half its maximum value (the HHD). HHD values for frequencies contaminated by line noise, e.g., $60 \mathrm{~Hz}$ and harmonics, are left blank.

\section{Figure 6.}

Population averages of the phase-lag index (PLI) and phase difference concentration (R). For each grid, the quantity (PLI or R) was averaged over all segments and channel pairs (blue bars), and a 95\% confidence interval (black whiskers) estimated via bootstrap sampling. The same quantities were averaged in the same way for surrogate data as described in the methods (red bars). Asterisks indicate significant differences between the measured quantities and surrogate quantities.

\section{Figure 7.}

Phase-lag index (PLI) and phase difference concentration (R) measured as a function of separation distance. For each grid, the quantity (PLI or R) was calculated by averaging results for all channel pairs separated by the same distance. The same calculations were performed on surrogate data as described in the methods. Shaded areas indicate confidence intervals around the average values. 


\section{References}

Alexander GE, DeLong MR, Strick PL. Parallel organization of functionally segregated circuits linking basal ganglia and cortex. Annu Rev Neurosci. 1986;9:357-81.

Amirikian B and Georgopoulos AP. Modular organization of directionally tuned cells in the motor cortex: is there a short-range order? Proc Natl Acad Sci U S A. 2003;100:12474-9.

Aoki F, Fetz EE, Shupe L, Lettich E, Ojemann GA. Increased gamma-range activity in human sensorimotor cortex during performance of visuomotor tasks. Clin Neurophysiol. 1999;110:524-37.

Ayzenshtat I, Meirovithz E, Edelman H, Werner-Reiss U, Bienenstock E, Abeles M, et al. Precise spatiotemporal patterns among visual cortical areas and their relation to visual stimulus processing. J Neurosci. 2010;30:11232-45.

Bansal AK, Truccolo W, Vargas-Irwin CE, Donoghue JP. Decoding 3D reach and grasp from hybrid signals in motor and premotor cortices: spikes, multiunit activity, and local field potentials. J Neurophysiol. 2012;107:1337-55.

Bansal AK, Vargas-Irwin CE, Truccolo W, Donoghue JP. Relationships among low-frequency local field potentials, spiking activity, and three-dimensional reach and grasp kinematics in primary motor and ventral premotor cortices. J Neurophysiol. 2011;105:1603-19.

Bedard C, Kroger H, Destexhe A. Modeling extracellular field potentials and the frequency-filtering properties of extracellular space. Biophys J. 2004;86:1829-42.

Berens P, Keliris GA, Ecker AS, Logothetis NK, Tolias AS. Comparing the feature selectivity of the gamma-band of the local field potential and the underlying spiking activity in primate visual cortex. Front Sys Neurosci. 2008;2:2.

Brinkmann BH, Bower MR, Stengel KA, Worrell GA, Stead M. Multiscale electrophysiology format: an open-source electrophysiology format using data compression, encryption, and cyclic redundancy check. Conf Proc IEEE Eng Med Biol Soc. 2009;2009:7083-6.

Bullock TH. Signals and signs in the nervous system: the dynamic anatomy of electrical activity is probably information-rich. Proc Natl Acad Sci U S A. 1997;94:1-6.

Bullock TH and McClune MC. Lateral coherence of the electrocorticogram: a new measure of brain synchrony. Electroencephalogr Clin Neurophysiol. 1989;73:479-98.

Bullock TH, McClune MC, Achimowicz JZ, Iragui-Madoz VJ, Duckrow RB, Spencer SS. EEG coherence has structure in the millimeter domain: subdural and hippocampal recordings from epileptic patients. Electroencephalogr Clin Neurophysiol. 1995a;95:161-77.

Bullock TH, McClune MC, Achimowicz JZ, Iragui-Madoz VJ, Duckrow RB, Spencer SS. Temporal fluctuations in coherence of brain waves. Proc Natl Acad Sci U S A. 1995b;92:11568-72.

Buzsaki G, Anastassiou CA, Koch C. The origin of extracellular fields and currents--EEG, ECoG, LFP and spikes. Nat Rev Neurosci. 2012;13:407-20.

Buzsaki G and Draguhn A. Neuronal oscillations in cortical networks. Science. 2004;304:1926-9.

Canolty RT and Knight RT. The functional role of cross-frequency coupling. Trends Cogn Sci. 2010;14:506-15.

Ch'ng YH and Reid RC. Cellular imaging of visual cortex reveals the spatial and functional organization of spontaneous activity. Front Integr Neurosci. 2010;4:20.

Chestek CA, Gilja V, Blabe CH, Foster BL, Shenoy KV, Parvizi J, et al. Hand posture classification using electrocorticography signals in the gamma band over human sensorimotor brain areas. J Neural Eng. 2013;10:026002.

Destexhe A, Contreras D, Steriade M. Spatiotemporal analysis of local field potentials and unit discharges in cat cerebral cortex during natural wake and sleep states. J Neurosci. 1999;19:4595-608.

Engel AK, Konig P, Gray CM, Singer W. Stimulus-Dependent Neuronal Oscillations in Cat Visual Cortex: Inter-Columnar Interaction as Determined by Cross-Correlation Analysis. Eur J Neurosci. 1990;2:588-606.

Fein G, Raz J, Brown FF, Merrin EL. Common reference coherence data are confounded by power and phase effects. Electroencephalogr Clin Neurophysiol. 1988;69:581-4.

Felleman DJ and Van Essen DC. Distributed hierarchical processing in the primate cerebral cortex. Cereb Cortex. 1991;1:1-47.

Fetz EE, Chen D, Murthy VN, Matsumura M. Synaptic interactions mediating synchrony and oscillations in primate sensorimotor cortex. J Physiol Paris. 2000;94:323-31.

Flinker A, Chang EF, Barbaro NM, Berger MS, Knight RT. Sub-centimeter language organization in the human temporal lobe. Brain Lang. 2011;117:103-9.

Freeman WJ and Barrie JM. Analysis of spatial patterns of phase in neocortical gamma EEGs in rabbit. J Neurophysiol. 2000;84:1266-78.

Fries P. Neuronal gamma-band synchronization as a fundamental process in cortical computation. Annu Rev Neurosci. 2009;32:209-24.

Gaona CM, Sharma M, Freudenburg ZV, Breshears JD, Bundy DT, Roland J, et al. Nonuniform High-Gamma (60-500 Hz) Power Changes Dissociate Cognitive Task and Anatomy in Human Cortex. J Neurosci. 2011;31:2091-100.

Georgopoulos A, Taira M, Lukashin A. Cognitive neurophysiology of the motor cortex. Science. 1993;260:47-52.

Guevara R, Velazquez JL, Nenadovic V, Wennberg R, Senjanovic G, Dominguez LG. Phase synchronization measurements using electroencephalographic recordings: what can we really say about neuronal synchrony? Neuroinformatics. 2005;3:301-14. 
Hwang EJ and Andersen RA. Brain control of movement execution onset using local field potentials in posterior parietal cortex. J Neurosci. 2009;29:14363-70.

Hwang EJ and Andersen RA. Spiking and LFP activity in PRR during symbolically instructed reaches. J Neurophysiol. 2012;107:836-49.

Jia X, Smith MA, Kohn A. Stimulus selectivity and spatial coherence of gamma components of the local field potential. J Neurosci. 2011;31:9390-403.

Katzner S, Nauhaus I, Benucci A, Bonin V, Ringach DL, Carandini M. Local Origin of Field Potentials in Visual Cortex. Neuron. 2009;61:35-41.

Kellis S, Greger B, Hanrahan S, House P, Brown R. Platinum microwire for subdural electrocorticography over human neocortex: Millimeter-scale spatiotemporal dynamics. Conf Proc IEEE Eng Med Biol Soc. 2011a:4761-5.

Kellis S, Greger B, Hanrahan S, House P, Brown R. Sensing millimeter-scale dynamics in cortical surface potentials for neural prosthetics. Conf Proc IEEE Sensors. 2011b:1823-6.

Kellis S, Miller K, Thomson K, Brown R, House P, Greger B. Decoding spoken words using local field potentials recorded from the cortical surface. J Neural Eng. 2010;7:056007.

Kellis SS, House PA, Thomson KE, Brown R, Greger B. Human neocortical electrical activity recorded on nonpenetrating microwire arrays: applicability for neuroprostheses. Neurosurg Focus. 2009;27:E9.

Kelly RM and Strick PL. Cerebellar loops with motor cortex and prefrontal cortex of a nonhuman primate. J Neurosci. 2003;23:8432-44.

Kim D-H, Viventi J, Amsden JJ, Xiao J, Vigeland L, Kim Y-S, et al. Dissolvable films of silk fibroin for ultrathin conformal biointegrated electronics. Nat Mater. 2010;9:511-7.

Kreiman G, Hung CP, Kraskov A, Quiroga RQ, Poggio T, DiCarlo JJ. Object selectivity of local field potentials and spikes in the macaque inferior temporal cortex. Neuron. 2006;49:433-45.

Leski S, Linden H, Tetzlaff T, Pettersen KH, Einevoll GT. Frequency dependence of signal power and spatial reach of the local field potential. PLoS computational biology. 2013;9:e1003137.

Leuthardt EC, Freudenberg Z, Bundy D, Roland J. Microscale recording from human motor cortex: implications for minimally invasive electrocorticographic brain-computer interfaces. Neurosurg Focus. 2009;27:E10.

Linden H, Tetzlaff T, Potjans TC, Pettersen KH, Grun S, Diesmann M, et al. Modeling the spatial reach of the LFP. Neuron. 2011;72:859-72.

Liu J and Newsome WT. Local field potential in cortical area MT: stimulus tuning and behavioral correlations. J Neurosci. 2006;26:7779-90.

Logothetis NK, Kayser C, Oeltermann A. In Vivo Measurement of Cortical Impedance Spectrum in Monkeys: Implications for Signal Propagation. Neuron. 2007;55:809-23.

Maynard EM, Nordhausen CT, Normann RA. The Utah Intracortical Electrode Array: A recording structure for potential braincomputer interfaces. Electroencephalogr Clin Neurophysiol. 1997;102:228-39.

Mehring C, Nawrot MP, de Oliveira SC, Vaadia E, Schulze-Bonhage A, Aertsen A, et al. Comparing information about arm movement direction in single channels of local and epicortical field potentials from monkey and human motor cortex. $\mathbf{J}$ Physiol Paris. 2004;98:498-506.

Menon V, Freeman WJ, Cutillo BA, Desmond JE, Ward MF, Bressler SL, et al. Spatio-temporal correlations in human gamma band electrocorticograms. Electroencephalogr Clin Neurophysiol. 1996;98:89-102.

Mitra P and Bokil H. Observed brain dynamics: Oxford University Press; 2008.

Mitzdorf U. Current source-density method and application in cat cerebral cortex: investigation of evoked potentials and EEG phenomena. Physiol Rev. 1985;65:37-100.

Mitzdorf U. Properties of the evoked potential generators: current source-density analysis of visually evoked potentials in the cat cortex. Int J Neurosci. 1987;33:33-59.

Mollazadeh M, Greenwald E, Thakor N, Schieber M, Cauwenberghs G. Wireless micro-ECoG recording in primates during reach-to-grasp movements. Conf Proc IEEE BioCAS. 2011:237-40.

Mountcastle VB. Modality and topographic properties of single neurons of cat's somatic sensory cortex. J Neurophysiol. 1957;20:408-34.

Mountcastle VB. The columnar organization of the neocortex. Brain. 1997;120 ( Pt 4):701-22.

Nordhausen CT, Maynard EM, Normann RA. Single unit recording capabilities of a 100 microelectrode array. Brain Res. 1996;726:129-40.

Nordhausen CT, Rousche PJ, Normann RA. Optimizing recording capabilities of the Utah Intracortical Electrode Array. Brain Res. 1994;637:27-36.

Panagiotides H, Freeman WJ, Holmes MD, Pantazis D. Behavioral states may be associated with distinct spatial patterns in electrocorticogram. Cogn Neurodyn. 2011;5:55-66.

Pei X, Leuthardt EC, Gaona CM, Brunner P, Wolpaw JR, Schalk G. Spatiotemporal dynamics of electrocorticographic high gamma activity during overt and covert word repetition. Neuroimage. 2011;54:2960-72.

Penfield W and Jasper H. Epilepsy and the Functional Anatomy of the Human Brain. South Med J. 1954;47:704.

Rakic P. Specification of cerebral cortical areas. Science. 1988;241:170-6.

Rakic P. Confusing cortical columns. Proc Natl Acad Sci U S A. 2008;105:12099-100. 
Ray S, Crone NE, Niebur E, Franaszczuk PJ, Hsiao SS. Neural Correlates of High-Gamma Oscillations (60-200 Hz) in Macaque Local Field Potentials and Their Potential Implications in Electrocorticography. J Neurosci. 2008;28:11526-36.

Salinas E and Sejnowski TJ. Correlated neuronal activity and the flow of neural information. Nat Rev Neurosci. 2001;2:539-50.

Schevon CA, Goodman RR, McKhann G, Jr., Emerson RG. Propagation of epileptiform activity on a submillimeter scale. J Clin Neurophysiol. 2010;27:406-11.

Schevon CA, Ng SK, Cappell J, Goodman RR, McKhann G, Jr., Waziri A, et al. Microphysiology of epileptiform activity in human neocortex. J Clin Neurophysiol. 2008;25:321-30.

Schevon CA, Trevelyan AJ, Schroeder CE, Goodman RR, McKhann G, Jr., Emerson RG. Spatial characterization of interictal high frequency oscillations in epileptic neocortex. Brain. 2009;132:3047-59.

Seyedhosseini M, Shushruth S, Davis T, Ichida JM, House PA, Greger B, et al. Informative features of local field potential signals in primary visual cortex during natural image stimulation. J Neurophysiol. 2014:jn. 00278.2014.

Shen B, Nadkarni M, Zappulla RA. Spectral modulation of cortical connections measured by EEG coherence in humans. Clin Neurophysiol. 1999;110:115-25.

Singer W and Gray CM. Visual Feature Integration and the Temporal Correlation Hypothesis. Annu Rev Neurosci. 1995;18:55586.

Slutzky MW, Jordan LR, Krieg T, Chen M, Mogul DJ, Miller LE. Optimal spacing of surface electrode arrays for brain-machine interface applications. J Neural Eng. 2010;7:26004.

Slutzky MW, Jordan LR, Miller LE. Optimal spatial resolution of epidural and subdural electrode arrays for brain-machine interface applications. Conf Proc IEEE Eng Med Biol Soc. 2008:3771-4.

Stacey WC, Kellis S, Greger B, Butson CR, Patel PR, Assaf T, et al. Potential for unreliable interpretation of EEG recorded with microelectrodes. Epilepsia. 2013;54:1391-401.

Stacey WC, Kellis S, Patel PR, Greger B, Butson CR. Signal distortion from microelectrodes in clinical EEG acquisition systems. J Neural Eng. 2012;9:056007.

Stam CJ, Nolte G, Daffertshofer A. Phase lag index: assessment of functional connectivity from multi channel EEG and MEG with diminished bias from common sources. Hum Brain Mapp. 2007;28:1178-93.

Stead M, Bower M, Brinkmann BH, Lee K, Marsh WR, Meyer FB, et al. Microseizures and the spatiotemporal scales of human partial epilepsy. Brain. 2010;133:2789-97.

Towle VL, Syed I, Berger C, Grzesczcuk R, Milton J, Erickson RK, et al. Identification of the sensory/motor area and pathologic regions using ECoG coherence. Electroencephalogr Clin Neurophysiol. 1998;106:30-9.

von der Malsburg C. Binding in models of perception and brain function. Curr Opin Neurobiol. 1995;5:520-6.

Wang W, Degenhart AD, Collinger JL, Vinjamuri R, Sudre GP, Adelson PD, et al. Human motor cortical activity recorded with Micro-ECoG electrodes, during individual finger movements. Conf Proc IEEE Eng Med Biol Soc. 2009:586-9.

Waziri A, Schevon CA, Cappell J, Emerson RG, McKhann GM, 2nd, Goodman RR. Initial surgical experience with a dense cortical microarray in epileptic patients undergoing craniotomy for subdural electrode implantation. Neurosurgery. 2009;64:540-5; discussion 5.

Wodlinger B, Degenhart AD, Collinger JL, Tyler-Kabara EC, Wei W. The impact of electrode characteristics on electrocorticography (ECoG). Conf Proc IEEE Eng Med Biol Soc. 2011:3083-6.

Xing D, Yeh CI, Shapley RM. Spatial spread of the local field potential and its laminar variation in visual cortex. J Neurosci. 2009;29:11540-9.

Zaveri HP, Williams WJ, Sackellares JC, Beydoun A, Duckrow RB, Spencer SS. Measuring the coherence of intracranial electroencephalograms. Clin Neurophysiol. 1999;110:1717-25. 

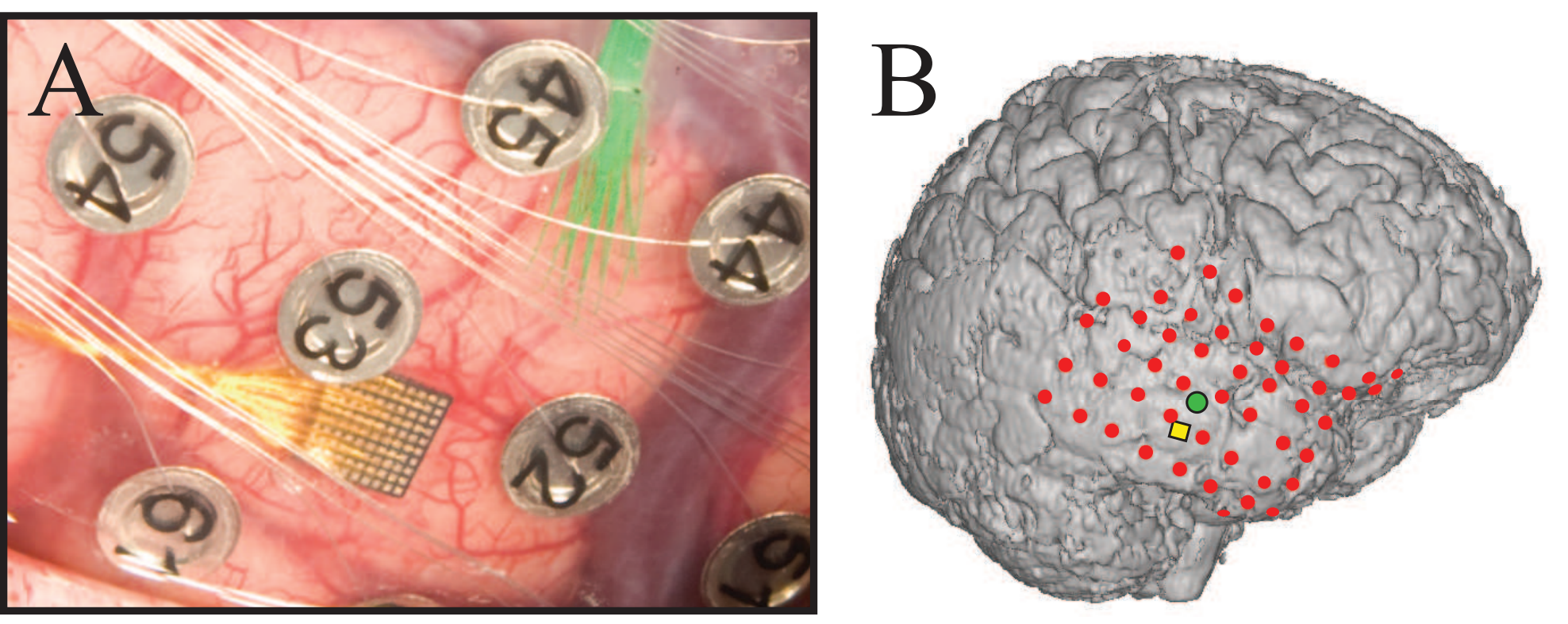

Figure 1
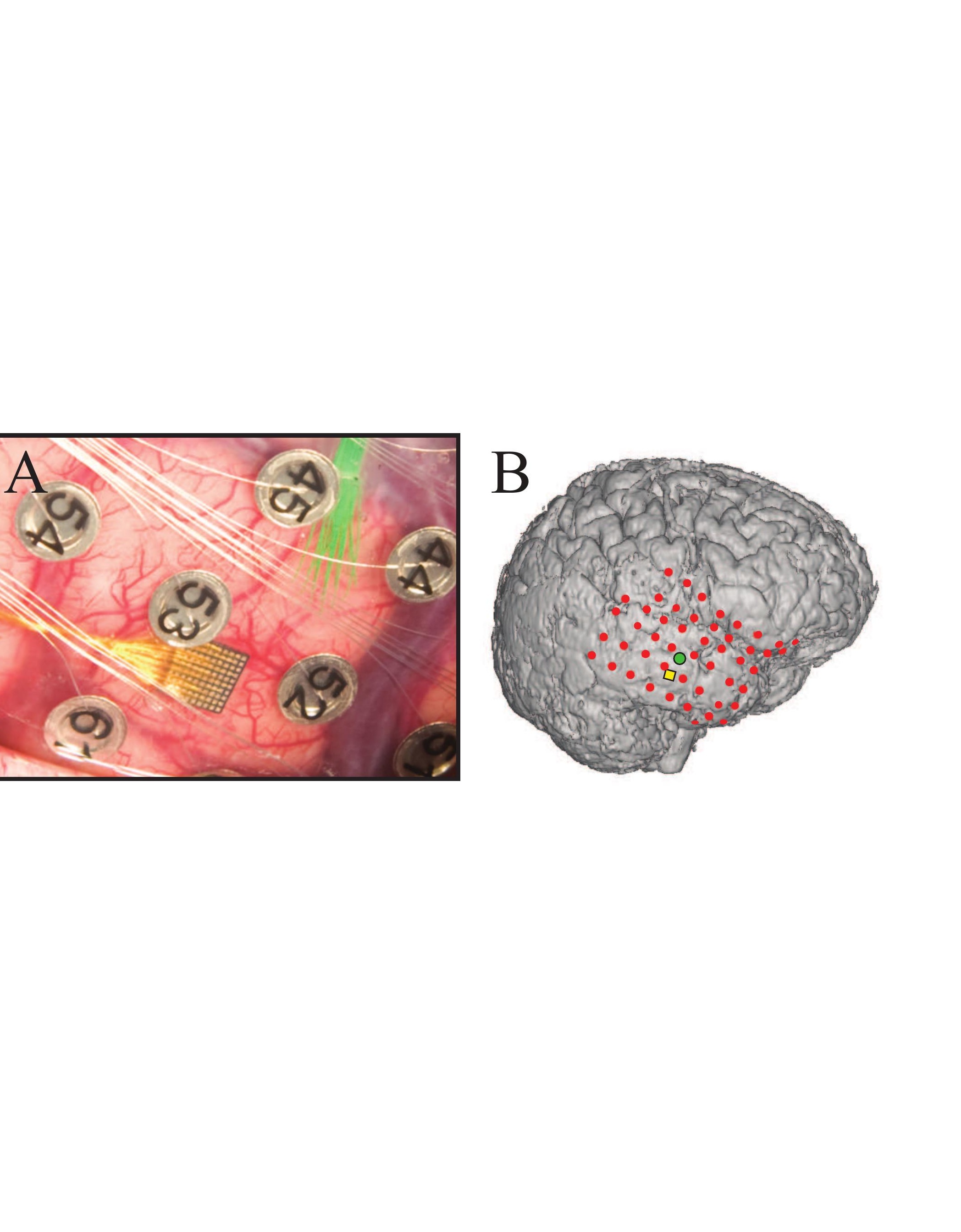
Figure $\mathbf{1}$

1.0

0.8
0.6
0.4
0.2
0.0
-0.2

$0 \quad 10$

20

30

40

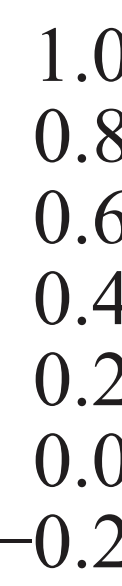

$0 \quad 10$
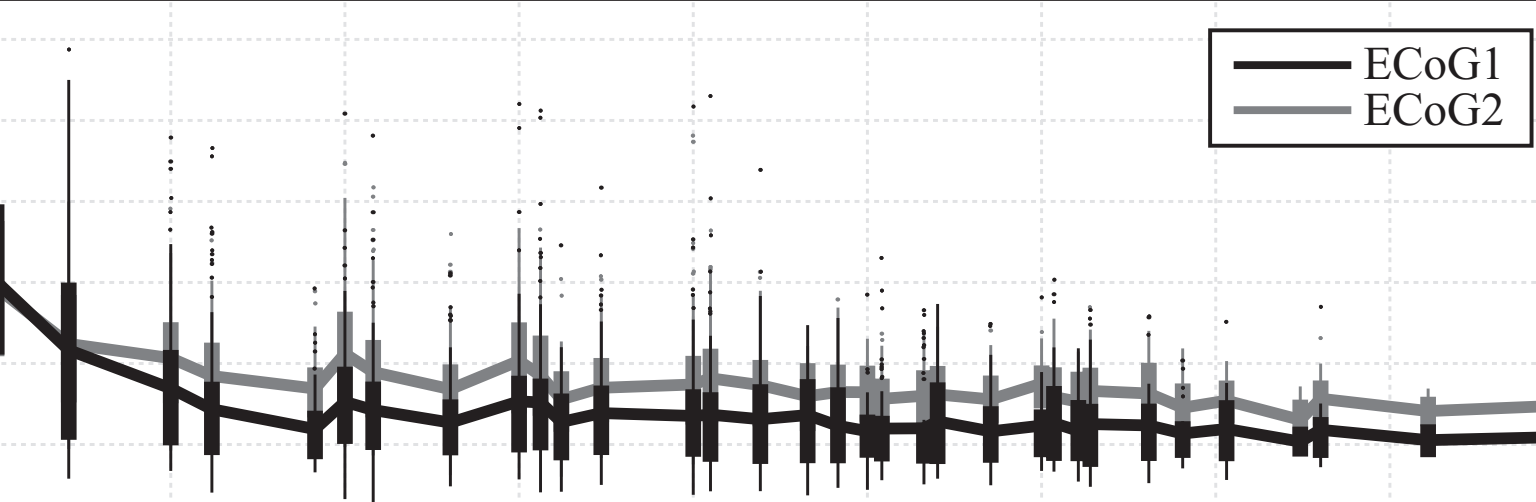

1.0
0.8
0.6
0.4
0.2
0.0

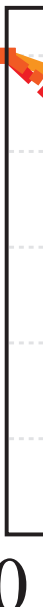

20

30

40

50

60

70

$\begin{array}{lll}80 & 90 & 100\end{array}$

.

1.0
0.8
0.6
0.4
0.2
0.0

0

23

4

5

separation distance $(\mathrm{mm})$

-ー- $\mu \mathrm{ECoG} 1$

$--m \mu \mathrm{ECoG} 2$ $-\mu \mathrm{ECoG} 3$ 
Figure 4
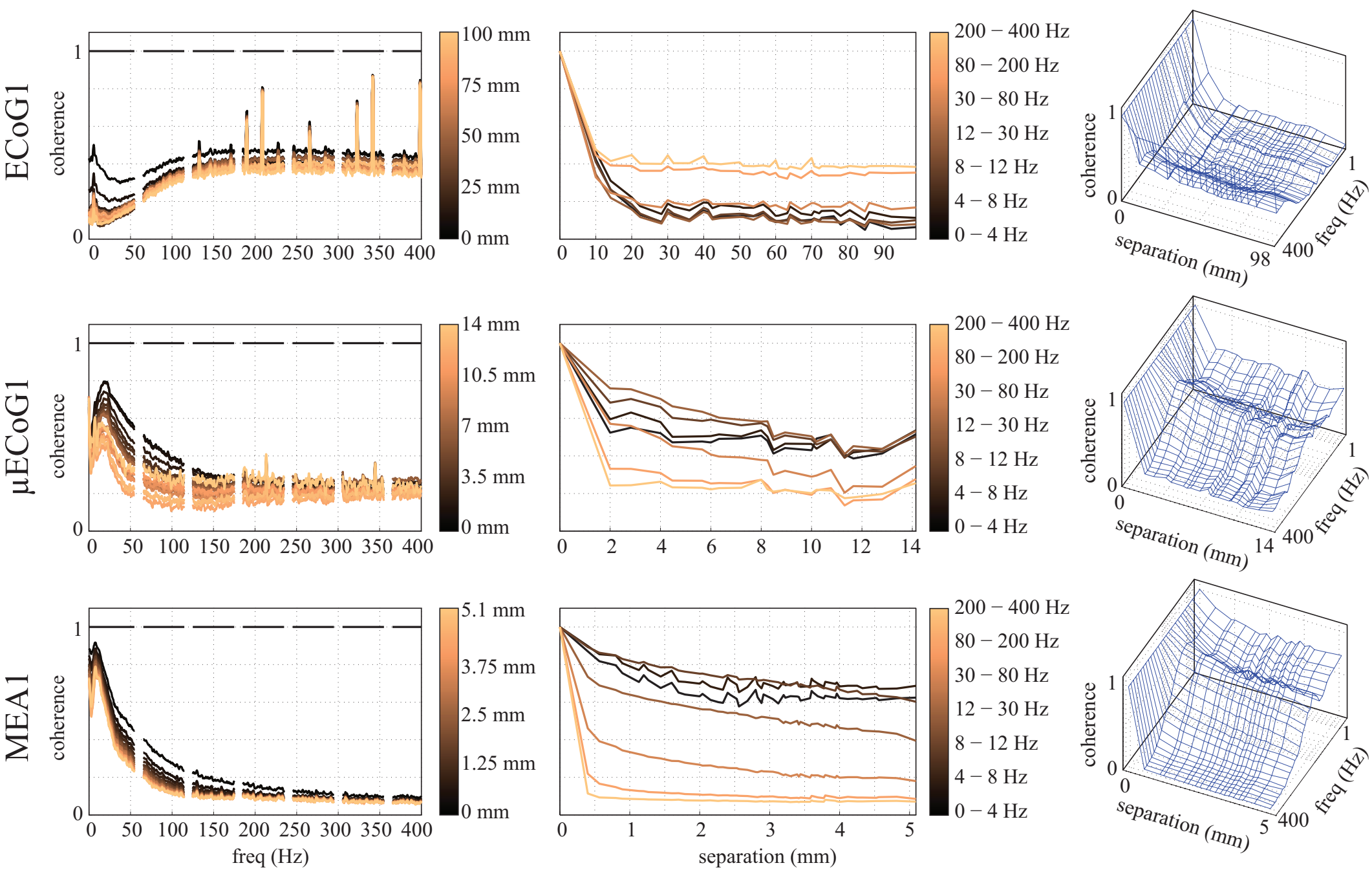


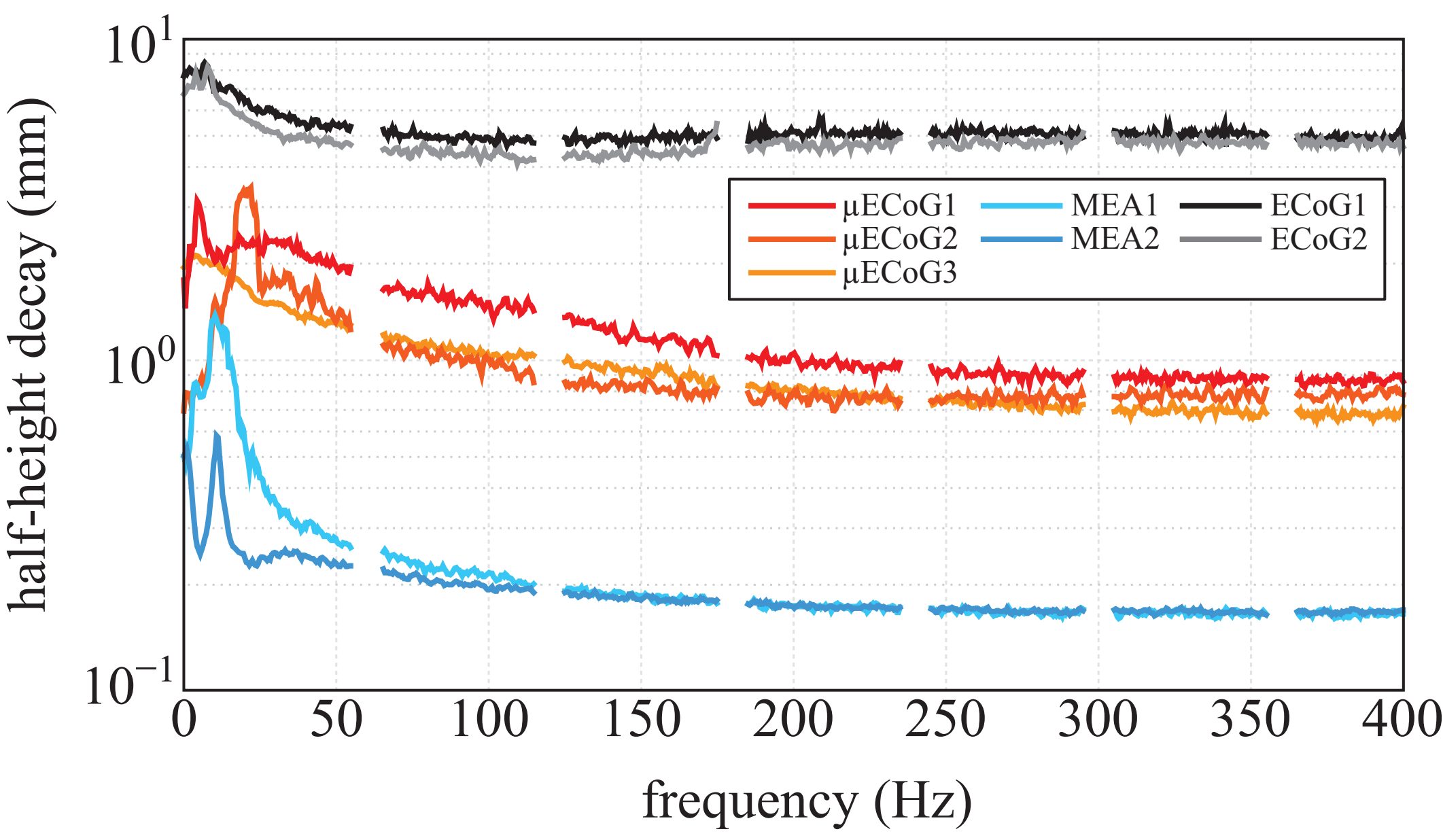

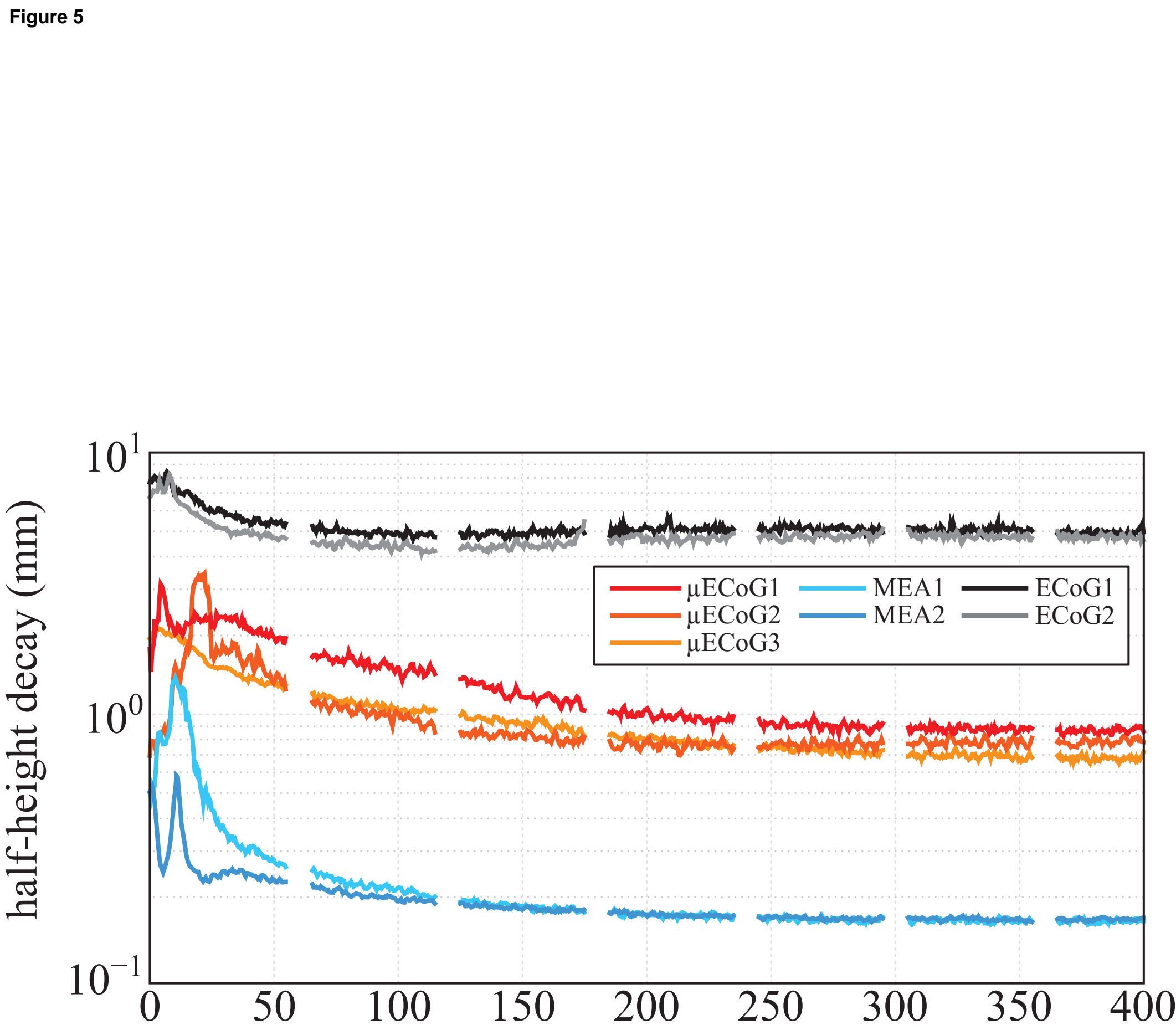
frequency $(\mathrm{Hz})$ 
Figure 6
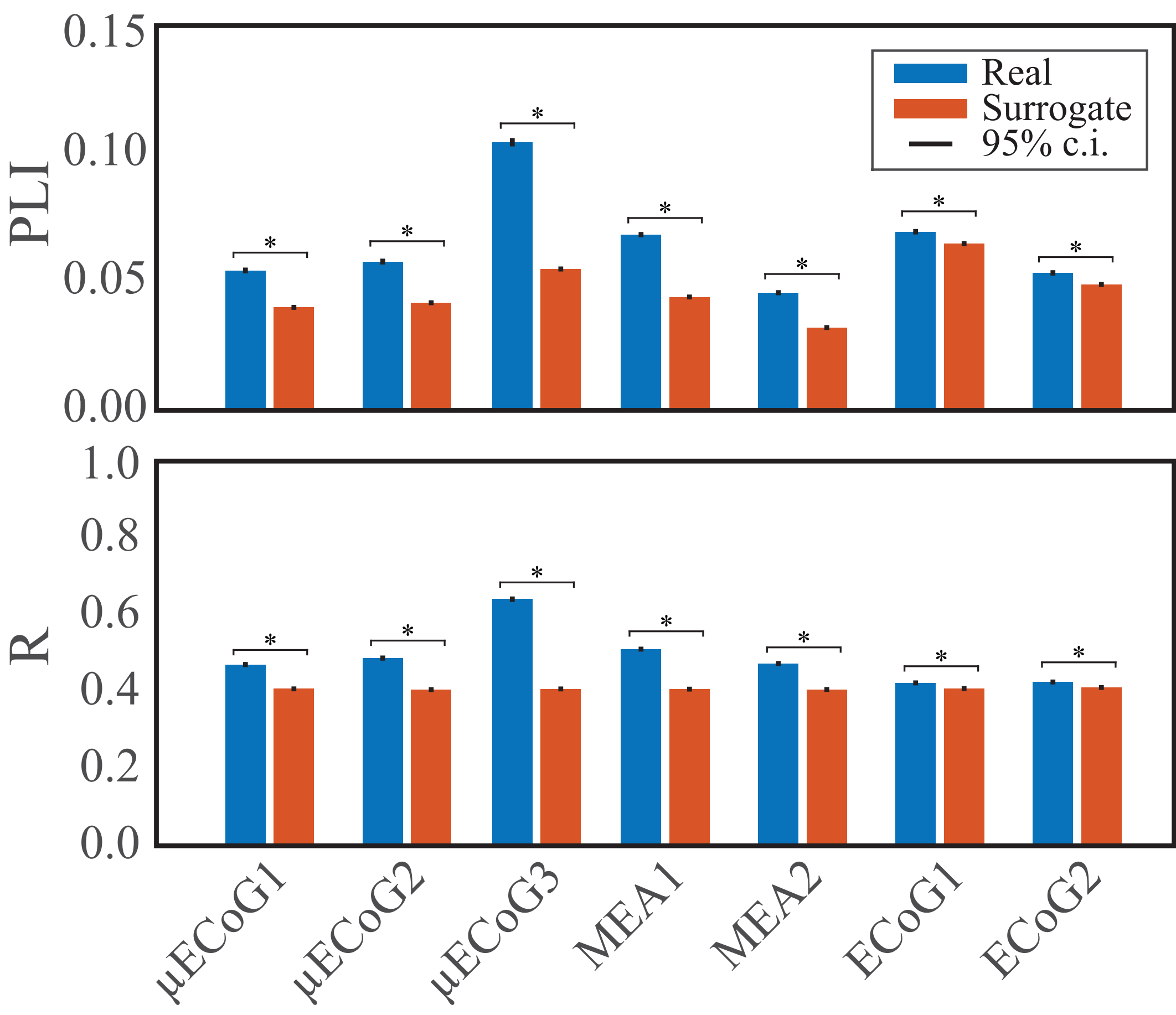
Figure 7
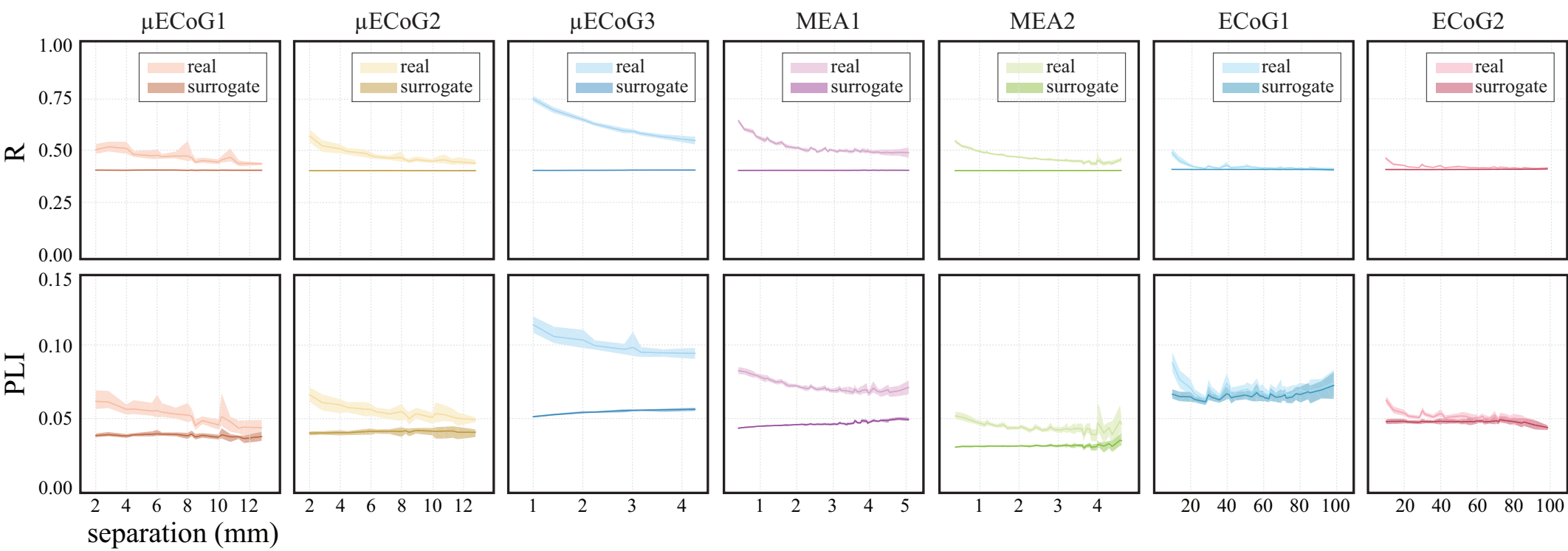
separation $(\mathrm{mm})$ 